\title{
Utilidad de la determinación de estradiol, progesterona, testosterona, sulfato de dehidroepiandrosterona, LH, FSH y prolactina para el ginecólogo.
}

\author{
Dr. Jaime Urdinola
}

El ginecólogo ha tenido que ver desde siempre con el diagnóstico $y$ el manejo de las anormalidades de la función menstrual.

Los conocimientos acerca de la esteroidogénesis ovárica y el desarrollo folicular, así como el de los mecanismos neuroendocrinos de control que participan en la secreción de gonadotropinas, se han aumentado considerablemente en los últimos años (1).

Estos procesos actúan concertadamente para producir una secuencia ordena-

Unidad de Investigación Clínica en Reproducción Humana.

Departamento de Ginecología y Obstetricia. Hospital Universitario "Lorencita V. de Santos".

Facultad de Medicina. Universidad Colegio Mayor de Nuestra Señora del Rosario, Bogotá, Colombia. da de eventos endocrinológicos y morfológicos que se conocen finalmente como el ciclo menstrual normal.

Tratando de entender la fisiología y la fisiopatología de las hormonas en Ginecología, estas fueron medidas clásicamente desde comienzos de este siglo en la sangre por medio del bioanálisis, midiendo la dosis-respuesta de diferentes órganos de animales. Varios principios fundamentales de la Endocrinología fueron establecidos mediante estos métodos.

Sin embargo, los métodos que utilizaban el bioanálisis, aunque adecuados para establecer cambios cualitativos, son relativamente imprecisos, no específicos dispendiosos en tiempo, costosos y requieren grandes cantidades de una muestra biológica para poder cumplir con los requerimientos de la investigación moderna y de la práctica clínica. 
Para evitar los problemas anteriormente mencionados, se dispone hoy en día de métodos analíticos con mayor sensibilidad y precisión, como el método del radioinmunoanálisis ( $R \mid A)$. Con este método relativamente sencillo, económico dentro de ciertos términos se determinan actualmente las hormonas en la institución.

Por medio del radioinmunoanálisis, en un sistema in-vitro y en base a una reacción inmunológica entre una sustancia a determinar o antígeno y un anticuerpo específicamente dirigido contra la misma, utilizando al mismo tiempo una sustancia análoga marcada con radioactividad como parámetro medible, se pueden cuantificar las concentraciones mínimas del antígeno buscado (2).

En la Tabla No. 1 aparecen los métodos analíticos físico-químicos y biológicos con la sensibilidad respectiva al frente. Debe enfatizarse que hasta el momento sólo por medio del RIA, es posible medir cantidades en el orden de los nanogramos (ng) y de los picogramos (pg) $\left(1 \mathrm{ng}=10^{-9} \mathrm{~g}, 1 \mathrm{pg}=10^{-12} \mathrm{~g}\right)$.

Tabla No. 1

\section{SENSIBILIDAD DE LOS METODOS ANALITICOS}

\begin{tabular}{|c|c|c|}
\hline \multirow{2}{*}{ Método } & \multicolumn{2}{|c|}{ Sensibilidad (ng) } \\
\hline & 100 & 10.000 \\
\hline $\begin{array}{l}\text { Espectrofotometría, } \\
\text { Absorción UV }\end{array}$ & 100 & 2.000 \\
\hline Fluoranálisis & 5 & 50 \\
\hline Doble dilucción con isótopos & $0.5-$ & 10 \\
\hline $\begin{array}{l}\text { Enzimoinmunoanálisis } \\
=\mathrm{EL} \text { IS A }\end{array}$ & $0,05-$ & 2,0 \\
\hline $\begin{array}{l}\text { Análisis + Radioactividad } \\
\text { C P B A }\end{array}$ & - & 10 \\
\hline $\begin{array}{l}\text { Enlace competitivo de proteínas } \\
\text { R A }\end{array}$ & $0,01-$ & 0,1 \\
\hline Análisis de receptores & & \\
\hline $\begin{array}{l}\text { R I A } \\
\text { Radioinmunoanálisis }\end{array}$ & 0,005 & 0,1 \\
\hline
\end{tabular}

Medir un picogramo equivaldría en forma figurada a determinar un cubo de azúcar de $5 \mathrm{~g}$ dentro de un lago de agua cuyas dimensiones serían $100 \mathrm{~m}$ de ancho, $100 \mathrm{~m}$ de largo y $100 \mathrm{~m}$ de profundidad.

La historia clínica detallada y el examen físico meticuloso en una paciente, dirigiendo la atención especialmente a los órganos "blanco" donde las hormonas ejercerán su efecto característico, siguen siendo los primeros pasos primordiales para orientar el diagnóstico de un problema de origen endocrino.

Pero a pesar de cumplir a cabalidad con estos requisitos, el ginecólogo en muchos casos no podrá llegar a un diagnóstico etiológico preciso, no pudiendo por lo tanto lograr el deseado tratamien. to causal y racional.

Esto es cierto en Ginecología, ya que una paciente puede presentar diferentes posibilidades diagnósticas con diversas posibilidades de tratamiento $y$ de pronóstico, teniendo en cuenta que muchos problemas endocrinos carecen de signos patognomónicos y sólo pueden diagnosticar por exclusión.

Tratando de solucionar este problema, el ginecólogo puede hoy en día hacer uso de las determinaciones hormonales, las cuales permitirán hacer un mejor diagnóstico y por ende un mejor tratamiento de su paciente.

Este trabajo presenta algunos conceptos acerca de la utilidad y de las limitaciones en la interpretación de algunas determinaciones hormonales realizadas por radioinmunoanálisis, de acuerdo a la experiencia clínica y de laboratorio obtenida en la institución.

La Tabla No. 2 muestra la estadística de las pruebas realizadas en el Laboratorio de Radioinmunoanálisis y Reproducción durante el lapso anotado. 
Tabla No. 2

Laboratorio de Radioinmunoanálisis

$$
\text { y Reproducción }
$$

\section{Departamento de Ginecología y}

Obstetricia Hospital Infantil Universitario "Lorencita V. de Santos"

Determinaciones Hormonales por

Radioinmunoanálisis -

26.6.1982 - 31.3.1985

\begin{tabular}{|lrrrrr|}
\hline Hormona & HC & Part & P H & Subtotal & \multicolumn{1}{c|}{} \\
\hline PRL & 736 & 484 & 135 & 1.353 & 19.6 \\
FSH & 496 & 312 & 87 & 895 & 12.2 \\
LH & 471 & 290 & 80 & 841 & 11.5 \\
BHCG & 383 & 247 & 97 & 727 & 10.0 \\
TSH & 376 & 172 & 80 & 628 & 8.6 \\
T4 & 252 & 183 & 90 & 525 & 7.1 \\
T3 & 268 & 144 & 74 & 486 & 6.6 \\
T3 UPTAKE & 78 & 56 & 13 & 147 & 2.0 \\
E 3 & 104 & 76 & 17 & 197 & 2.6 \\
P 4 & 79 & 49 & 41 & 169 & 2.3 \\
T & 161 & 106 & 41 & 308 & 4.2 \\
DHEAS & 53 & 49 & 14 & 116 & 1.5 \\
17 OH-P & 43 & 23 & 9 & 75 & 1.0 \\
ACTH & 12 & 8 & - & 20 & 0.2 \\
Cortisol & 48 & 20 & 3 & 71 & 0.9 \\
STH & 105 & 56 & - & 161 & 2.2 \\
Insulina & 64 & 28 & 17 & 109 & 1.4 \\
Estradiol & 13 & 6 & 6 & 25 & 0.3 \\
PTH & - & - & - & - & - \\
LPH & 3 & 1 & 12 & 16 & 0.2 \\
T4NN & 55 & 35 & - & 90 & 1.2 \\
TSH-NN & 152 & 152 & - & 304 & 4.1 \\
Renina & 6 & 3 & - & 9 & 0.1 \\
Somatomedina & 6 & - & - & 6 & 0.1 \\
Gastrina & 3 & 7 & - & 10 & 0.1 \\
\hline Total & 3.976 & 2.507 & 816 & 7.299 & 100.0 \\
\hline
\end{tabular}

Diferentes hormonas que pueden presentar variaciones dia a dia influencian el ciclo menstrual de la mujer.

Por esta razón, es deseable en muchas ocasiones estudiar más de una hormona para poder llegar a establecer conclusiones más reales.

Pero sólo el conocimiento de los prin- cipios básicos y la experiencia del clínico, le permitirán a este llegar a determinar el número y la frecuencia de determinaciones hormonales necesarias para el manejo adecuado de una paciente.

El ovario se expresa a través de su actividad secretora de diferentes sustancias. Esta puede evaluarse, encontrándola normal o patológica, al determinar los productos principales de la esteroidogénesis ovárica, los estrógenos, la progesterona o los andrógenos.

Si se determinan las hormonas proteicas como las gonadotropinas o la prolactina, puede evaluarse la actividad de la hipófisis anterior.

Es muy importante contar con el criterio de reproducibilidad para los resultados de laboratorio, ya sea que la muestra a analizar tenga propósitos de diagnóstico o de investigación. En este sentido es esencial disponer de un manejo estandarizado de las muestras dentro del laboratorio.

Se anota a continuación el procedimiento que se sigue y que es adaptable para las diferentes determinaciones hormonales que se procesan dentro del laboratorio.

En general deben tomarse por lo me nos $10 \mathrm{ml}$ de sangre, los cuales son colo cados inmediatamente en un tubo de vidrio. Después de 15 a 30 minutos aproximadamente, los tubos se centrifugan a $800 \mathrm{~g}(1600$ a 2000 RPM en condiciones locales) durante 10 minutos a temperatura ambiente.

Una vez la sangre total ha sido centrifugada, el plasma es removido y colocado en otro tubo de vidrio $y$ congelado entonces a por lo menos $-20^{\circ} \mathrm{C}$, hasta que las determinaciones lleguen a ser realizadas.

No se usa la anticoagulación en forma rutianria, sólo en ciertos casos especiales. 
Se sabe que no es conveniente el uso de heparina cuando se toman muestras para determinar el T3 Uptake que permitirá posteriormente calcular el T4 normalizado o libre.

Así mismo, no debe usarse EDTA para muestras de testosterona libre, ACTH o posiblemente de LH o FSH, aunque parezca ser conveniente en general para los esteroides (3).

Ciertas determinaciones requieren una toma múltiple de muestras, para eliminar las variaciones que ocurren con el tiempo como puede ser el caso de la prolactina, las gonadotropinas o en algunos casos la testosterona.

En la hoja para registrar la muestra de la paciente debe anotarse su nombre, si no es obvio se debe indicar el sexo, la edad, el diagnóstico presuntivo, la medicación sobre todo hormonal que la paciente haya estado tomando durante el último mes, la fecha y hora en que se tomó la muestra así como la fecha de la última menstruación para poder calcular el día del ciclo menstrual, contando como primero el día que comenzó el sangrado.

Para el caso de la toma múltiple de muestras, la muestra debe tomarse a los 0,30 y 60 minutos. A los 0 y 30 minutos es adecuado para las pacientes difíciles, sin embargo la primera forma anotada es mejor.

En caso de tener dificultades para obtener la muestra de sangre, puede usarse la Tabla No. 3 como una guía, que aparece a continuación.

Los datos que aquí se presentan, se refieren a la determinación de las siguientes hormonas en condiciones basales (sin estimulación): $E_{2}$.

Estrógenos: Midiendo el estradiol o
Tabla No. 3

\section{GUIA PARA LAS MUESTRAS DE LAS DETERMINACIONES HORMONALES}

\begin{tabular}{|lccc|}
\hline Hormona & $\begin{array}{c}\text { Cantidad minima } \\
\text { suero para RIA }\end{array}$ & $\begin{array}{l}\text { Cantidad deseada } \\
\text { de suero }(+)\end{array}$ & Muestra múltiple \\
\hline$E_{2}$ & $0.5 \mathrm{ml}$ & $1.0 \mathrm{ml}$ & - \\
$\mathrm{P}_{4}$ & $0.5 \mathrm{ml}$ & $1.0 \mathrm{ml}$ & - \\
$\mathrm{T}$ & $0.5 \mathrm{ml}$ & $1.0 \mathrm{ml}$ & $-/+$ \\
DHEAS & $0.5 \mathrm{ml}$ & $1.0 \mathrm{ml}$ & - \\
LH & $1.0 \mathrm{ml}$ & $2.0 \mathrm{ml}$ & + \\
FSH & $1.0 \mathrm{ml}$ & $2.0 \mathrm{ml}$ & + \\
PRL & $0.5 \mathrm{ml}$ & $1.0 \mathrm{ml}$ & + \\
\hline
\end{tabular}

$(+)=$ multiplicando por 2 se obtiene la cantidad de sangre

Progesterona: Denominada como $\mathrm{P}_{4}$.

Andrógenos: A través de la cuantificación de testosterona o $T$, y del sulfato de dehidroepiandrosterona o DHEAS.

Gonadotropinas: La hormona folículoestimulante o folitropina o $\mathrm{FSH}$ y la hormona luteinizante o lutropina o LH.

Prolactina: Cuya abreviatura internacional es la de PRL.

Teniendo en cuenta las diferentes variaciones de las hormonas durante el ciclo menstrual normal, se cita a las pacientes para la toma de muestras en la fase folicular, entre los días 5o. a 8o. del ciclo.

Se evita de esta manera la interpretación equivocada de un aparente hipergonadotropismo al coincidir la determinación con el pico ovulatorio de gonadotropinas o los resultados anormalmente altos en forma aparente del estradiol coincidentes con el pico del mismo que precede al de las gonadotropinas, esto a manera de ejemplo.

En la Tabla No. 4, que aparece a continuación, se detallan los valores considerados como normales dentro de la institución. Posteriormente se hace referencia específica a cada una de las hormonas. 
Tabla No. 4

\section{VALORES NORMALES}

\begin{tabular}{|c|c|c|c|c|}
\hline \multirow[t]{2}{*}{ Estado Clínico } & \multicolumn{3}{|l|}{ Hormonas } & \multirow[b]{2}{*}{ DHEAS } \\
\hline & $E_{2}$ & $P_{4 t}$ & $\mathbf{T}$ & \\
\hline & $\mathrm{pg} / \mathrm{ml}$ & $\mathrm{ng} / \mathrm{ml}$ & $\mathrm{ng} / \mathrm{ml}$ & $\mu \mathrm{g} / \mathrm{dl}$ \\
\hline Fase folicular & $25-75$ & $\leqslant 1$ & $0.2-0.9$ & $70-340$ \\
\hline Pico ovulatorio & $200-600$ & - & $0.2-0.9$ & $70-340$ \\
\hline \multirow[t]{2}{*}{ Fase lútea } & $100-300$ & $5-20$ & $0.2-0.9$ & $70-340$ \\
\hline & \multicolumn{2}{|l|}{$\begin{array}{l}\mathrm{LH} \\
\mathrm{mUl} / \mathrm{ml}\end{array}$} & $\begin{array}{l}\text { FSH } \\
\mathrm{mUI} / \mathrm{ml}\end{array}$ & $\begin{array}{l}\mathrm{PRL} \\
\mathrm{ng} / \mathrm{ml}\end{array}$ \\
\hline Mujer adulta normal & $5-20$ & & -20 & $<20$ \\
\hline Pico ovulatorio & \multicolumn{2}{|l|}{$\begin{array}{l}3 \text { veces } \\
\text { nivel basal }\end{array}$} & $\begin{array}{l}\text { veces } \\
\text { ivel basal }\end{array}$ & \\
\hline Hipogonadotropismo & \multicolumn{2}{|c|}{$<5$} & $<5$ & \\
\hline Hipergonadorotpismo & \multicolumn{2}{|c|}{$>25$} & $>40$ & \\
\hline
\end{tabular}

\section{ESTROGENOS}

El estradiol $\left(E_{2}\right)$ es el principal esteroide secretado por los folículos en maduración.

Los niveles periféricos del mismo comienzan a ascender entre los días 5 a 7 del ciclo, una vez que el proceso de selección del folículo dominante ha ocurrido (4).

El folículo preovulatorio maduro produce cantidades cada vez mayores de $E_{2}$, las cuales alcanzan un pico 24 a 36 horras aproximadamente antes de la ovulación, induciendo de esta manera el pico ovulatorio de la $\mathrm{LH}(5)$.

Después de la ovulación, la elevación nuevamente del estradiol circulante desde la fase lútea temprana, se asocia con una declinación de los niveles de progesterona $\left(\mathrm{P}_{4}\right)$, contribuyendo de esta ma nera a la luteolisis (6).

Desde el punto de vista teórico, determinar el $E_{2}$ en sangre podría ser útil, sin embargo en la práctica esto no es así por varias razones.

El rango de las concentraciones normales del $E_{2}$ es muy amplio a lo largo del ciclo, como puede apreciarse en la Tabla No. 4, mostrando valores muy bajos durante la fase proliferativa temprana del ciclo menstrual, similares inclusive a aquellos encontrados en el varón. Pero esta condición de hipoestrogenismo, con valores inclusive por debajo de lo normal en una mujer, es fácilmente detectable por la clínica sin la ayuda del laboratorio, en forma más rápida y más económica.

Se puede apreciar su efecto en órganos fácilmente accesibles al ginecólogo, como la mucosa vaginal y el moco cervical y al respecto existen métodos perfectamente estandarizados y con los cuales se posee una amplia experiencia $(7,8)$.

En niñas prepúberes o en mujeres climatéricas, los tumores productores de estrógenos producen generalmente manifestaciones clínicas que pueden alertar al médico. En los casos equívocos sin embargo, una determinación de $E_{2}$ puede ayudar a aclarar el diagnóstico.

En los casos de pubertad precoz, la determinación del $E_{2}$, así como de la DHEAS y de las gonadotropinas, pueden ayudar a establecer el diagnóstico y el pronóstico.

Donde si tiene cabida ineludible hasta el momento, a pesar de contar hoy en día con la ayuda de la ecografía o ultrasonografía, la determinación serieda del $E_{2}$, es dentro del programa de inducción de la ovulación con gonadotropinas, ya sea con HMG-hCG (gonadotropinas de mujer menopáusica-gonadotropina coriónica humana) o con FSH pura. La dosis óptima de gonadotropinas administrada, permite una maduración folicular adecuada y por consiguiente la ovulación exitosa. Las dosis menores pueden conllevar a un fallo de la misma, así como las dosis excesivas pueden conllevar a un embarazo múltiple o a un sindrome de hiperestimulación ovárica (9). 


\section{PROGESTERONA}

La ovulación es la culminación de una serie de eventos sincronizados donde han participado el ovario y el sistema nervioso central.

La progesterona o $\mathrm{P}_{4}$ es una de las hormonas producidas por el cuerpo lúteo. Durante la fase folicular sus niveles en sangre prácticamente no son detectables, como puede apreciarse en la Tabla No. 4.

Se puede detectar un aumento en su producción, en la vena eferente del ovario portador del folículo dominante, desde 24 a 48 horas antes de la ovulación y uno mayor que ocurre durante el día del pico de la LH, de gran importancia fisiológica para aumentar la acción de retroalimentación positiva del $E_{2}$ e inducir el pico combinado de mitad del ciclo de la LH y de la $\operatorname{FSH}(10,11)$.

Los niveles de $\mathrm{P}_{4}$ ascienden marcadamente después de la ovulación, alcanzando su valor máximo aproximadamente 8 días después del pico de $\mathrm{LH}$, declinando desde entonces gradualmente $y$ regresando a sus concentraciones basales al comienzo de la siguiente menstruación (12).

La determinación de $\mathrm{P}_{4}$ proporciona evidencia acerca de la ocurrencia bioquímica de la ovulación.

Sin embargo, hay otros métodos que pueden proporcionar esta información, como la curva de temperatura basal con todas sus limitaciones, las determinaciones seriadas de moco cervical y en forma inmediata la ecografía o ultrasonografía, permitiendo esta última el poder descartar el síndrome de folículo no roto.

La biopsia de endometrio puede considerarse también como un bioanálisis muy preciso al respecto, añadiendo a la información sobre la ovulación, la de si el endometrio se encuentra o no en fase, permitiendo descartar el síndrome de fase lútea insuficiente (13).

Puede ser de utilidad en aquellas pacientes bajo seguimiento en quienes no es posible obtener la medición de la temperatura basal o quienes no pueden ser sometidas a biopsias de endometrio a repetición.

\section{ANDROGENOS}

Es importante considerar la determinación de los andrógenos dentro del estudio de mujeres que consultan por hirsutismo, virilización, piel grasosa, sudoración aumentada, acné, menstruaciones irregulares o infertilidad, todas estas manifestaciones de hiperandrogenismo. Otros efectos metabólicos generales pueden incluir la resistencia periférica a la insulina y obesidad asociada. No se debe escapar tampoco la bien conocida asociación con el síndrome de ovarios poliquísticos u ovario androgénico o anovulatorio.

El hiperandrogenismo es una de las endocrinopatías más comunes en la mujer, pudiendo conllevar a un síndrome de anovulación crónica y representando en la consulta de la institución un poco más del $40 \%$ de las pacientes atendidas.

Bajo circunstancias normales, la producción de esteroides sexuales por parte de la glándula suprarrenal es menos significativa que la producción de andrógenos y estrógenos por parte de las gónadas. Normalmente el $50 \%$ de la producción de testosterona se obtiene a través de la conversión periférica a partir de androstenediona, 25\% se secreta por parte del ovario y el $25 \%$ restante de la glándula suprarrenal.

En el ciclo menstrual normal, la acumulación de tejido estromal la mitad del ciclo puede aumentar los niveles circulantes de androstenediona y testosterona durante la ovulación. 
Determinar la $\mathrm{T}$ y la DHEAS tienen por objeto diferenciar la hiperandrogenemia no tumoral de la tumoral, ya que esta última requiere de cirugía (14).

La paciente con hipernadrogenemia de origen no tumoral, tendrá valores que no exceden de $2 \mathrm{ng} / \mathrm{ml}$ de $T$, mientras que en aquellas en quienes se encuentren valores superiores será imperativo descartar la presencia de un tumor.

La DHEAS ha sustituido la determinación de los 17-cetosteroides en orina de 24 horas para la evaluación del hirsutismo, ya que se deriva casi exclusivamente de la glándula suprarrenal, no necesitando de correcciones en relación al peso corporal, la excreción de creatinina. Como se excreta en altas concentraciones y posee una vida media larga, no se espera por parte de ella que presente variaciones durante el ciclo menstrual.

Si se obtienen valores dentro de los límites normales, puede excluirse prácticamente un problema a nivel Suprarrenal, estableciéndose el diagnóstico de producción androgénica excesiva por parte de los ovarios.

El problema clínico se presenta en las pacientes con ovario poliquístico o anovulatorio donde puede presentarse una moderada elevación de la DHEAS, así como en asociación con hiperprolactinemia (15).

En el caso raro de una paciente con niveles de DHAS por encima de 700ug/ dl, debe descartarse la presencia de un tumor o de una hiperplasia suprarrenal.

\section{GONADOTROPINAS}

La hipófisis anterior está controlada por muchos factores, en forma positiva - negativa por los esteroides sexuales, por su respuesta a las hormonas liberadoras $y$ depende de la integridad y con- tinuidad funcional $y$ anatómica del eje hipotálamo-hipófisis.

La FSH y la LH representan por lo tanto los productos finales de su actividad y reactividad y no necesariamente el hiper o el Hipogonadotropismo denotan una disfunción primaria de la parte anterior de la glándula.

En la mujer normal la LH y la FSH son secretadas en forma pulsátil por la influencia de la luliberina o GnRH o LHRH, siendo este patrón más pronunciado en la LH que en la FSH (16).

La utilidad clínica de su determinación puede apreciarse en los ejemplos siguientes:

Debe tenerse muy en cuenta el día del ciclo en que se tomó la muestra, ya que los valores elevados de las gonadotropinas pueden indicar un fallo ovárico, indicándonos una disgenesia gonadal, un fallo ovárico prematuro, una menopausia precoz, el climaterio o una castración, pero pueden también ser coincidentes con el pico ovulatorio (17).

Es normal en el período cerca a la menopausia que los niveles de $\mathrm{FSH}$ comiencen a elevarse aun antes de que el sangrado haya cesado. Valores por encima de $40 \mathrm{mUl}-\mathrm{ml}$ para esta hormona son compatibles con la menopausia.

En el síndrome de ovario poliquístico o anovulatorio, permiten reconocerlo al encontrar altas concentraciones de LH, con FSH normal o baja (18). EI establecimiento de una relación LH/FSH mayor a 2 es hoy uno de los criterios bioquímicos utilizados para la confirmación de este diagnóstico.

Valores por debajo del límite inferior normal pueden encontrarse en entidades como la anorexia nervosa o en la amenorrea de origen hipotalámico, siendo nece sario de todas maneras distinguir si el 
problema se origina en el hipotálamo o en la hipófisis, como podría ser el caso de un síndrome de Sheehan (19).

\section{PROLACTINA}

Esta es otra de las hormonas secretadas por la hipófisis anterior, aunque su secreción está mediada en parte por la dopamina (20).

La hiperprolactinemia como hallazgo bioquímico puede estar asociada con trastornos del ciclo menstrual, insuficiencia del cuerpo lúteo, anovulación, con el denominado sindrome de amenorrea-galactorrea y con infertilidad.

No debe olvidarse que la TRH puede aumentar la secreción de PRL. Es importante entonces en los casos de sospecha de una hiperprolactinemia, determinar simultáneamente la TSH para poder confirmar o descartar la presencia de un hipotiroidismo de origen central.

El objetivo principal al encontrar una hiperprolactinemia, es el de poder ex-

\section{BIBLIOGRAFIA}

1. FRITZ MA., SPEROFF L: The endocrinology of the menstrual cycle: the interaction of folliculogenesis and neuro endocrine mechanisms. Fertil Sterir 38:

2. BERSON SA, YALOW RS: General Radioinmunoassay, in Berson SA, Yalow RA (eds): Methods in Investigative and Diagnostic Endocrinology: I. General Methodology. Amsterdam, North Holland Publishing Co, 1973, pp. 84-120.

3. COLSTON WENTZ A, GIVENS JR, ANDERSON RH, COHEN BM: Specimen Handling, in Manual of Gynecologic Endrocrinology and Infertility. Baltimore, Williams and Wilkins, 1979, pp. 99-100. cluir la presencia de un tumor a nivel de los lactotropos hipofisiarios o prolactinoma (21). Aunque se acepta hoy en día que niveles por encima de los 100 $\mathrm{ng} / \mathrm{ml}$ se asocian con este tipo de tumores, es esencial por lo menos descartar en forma rutinaria el prolactinoma por otros medios como la placa simple de silla turca.

En la mujer adulta, la prolactina en forma basal presenta variaciones episódicas o pulsátiles de baja amplitud (22). Por esta razón en la institución se toma la muestra en forma de pool a los 0 , 30 y 60 minutos, rutinariamente.

La frecuencia de hiperprolactinemia en el síndrome de ovario poliquístico o anovulatorio varía entre el 13 y el $41 \%$. Sin embargo, cuando se toman muestras múltiples cada 20 minutos durante 4 veces, en aquellas pacientes con hiperprolactinemia, la frecuencia se ha disminuido hasta el $17 \%$, logrando de esta manera una interpretación más acorde con la realidad de la hiperprolactinemia (23).

4. DIZEREGA GS, MARUT EL, TURNER CK, HODGEN GD: Asymmetrical ovarian function during recruitment and selection of the dominant follicle in the menstrual cycle of the rhesus monkey. J. Clin. Endocrinol Metab 51: 698, 1980.

5. PAUERSTEIN CJ, EDDY CA, CROXAT. TO HD, HESS R, SILER-KHODR TM, CROXATTO HB: Temporal relationships of estrogen, progesterone, and luteinizing hormone levels to ovulation in woman and infrahuman primates. Am J. Obstet. 130, 876, 1978.

6. KARSCH JF, KREY LC, WEICK RF, DIERSCHKE DJ, KNOBIL E: Functional luteolysis in the rhesus monkey: the role of estrogen. Endocrinology 92: 1148, 1973. 
7. WHO Special Programme of Research, Development and Research Training in Human Reproduction: Laboratory Manual for the Examination of Human Semen and Semen-Cervical Mucus Interaction. Singapore, Press Concern, 1980.

8. JONES JR. HW, JONES GS: Cyclical Citology and Histology of the Genital Tract, in JONES JR. HW, JONES GS: Novak's Textbook of Gynecology, Tenth Edition. Baltimore, Williams and Wilkins, 1981, pp. 68-103.

9. HANING RV, AUSTIN CHW, CARLSON IH, KUZMA DL, SHAPIRO SS, ZWEIBEL WJ: Plasma estradiol is superior to ultrasound and urinary estriol glucuronide as a predictor of ovarian hyperstimulation during induction of ovulation with menotropins. Fertil Steril 40: 31, 1983.

10. MOGHISSI KS, SYNER FN, EVANS TN: A composite picture of the menstrual cycle. Am J. Obstet. Gynecol. 114: 405, 1972.

11. TERASAWA E, RODRIGUE-Y-SIERRA JF, DIERSCHKE DJ, BRIDSON WE, GOY RW: Positive feedback effect of progesterone on luteiniying hormone (LH) release in cyclic female rhesus monkeys: LH response occurs in two phases. J Clin Endocrinol Metab 51: 1245, 1980.

12. DIZEREGA, HODGEN GD: The interovarian progesterone gradient: a spatial and temporal regulator of folliculogenesis in the primate ovarian cycle. J.Clin. Endocrinol Metab 54: 495, 1982.

13. JONES GS, MADRIGAL-CASTRO V: Hormonal findings in association with abnormal corpus luteum function in the human: the luteal phase defect. Fertil 21: 1, 1970.

14. SCHWARTZ U, MOLTZ L, BROTHERJ, HAMMERSTEIN J: The diagnostic value of plasma free testosterone in non- tumorous and tumorous hypernadrogenism. Fertil Steril 40: 66, 1983.

15. LOBO RA, KLETSKY OA, KAPTEIN EM, GOEBELSMANN U: Prolactin modulation of dehydroepiandrosterone sulfate secretion. Am. J. Obstet. Gynecol. 138: $632,1980$.

16. KNOBIL E: The neuroendocrine control of the menstrual cycle. Recent Prog Horm Res 36: 53, 1980.

17. REBAR RW, ERIKSON GF, YEN SSC: Idiopathic premature ovarian failure: clinical and endocrine characteristics. Fertil Steril 37: 35, 1982.

18. DE VANE GW, CZEKALA NM, JUDD HL, YEN SSC: Circulating gonadotropins, estrogen and androgens in policystic ovarian disease. Am J Obstet Gynecol 121: 496, 1975.

19. WARREN MP, VAN DE WIELE RL: Clinical and metabolic features of anorexia nervosa. Am. J. Obstet. Gynecol.117: 435, 1973.

20. KAMBERY IA, MICAL RS, PORTER JC: Effect of anterior pituitary perfusion and intravenous injection of catecholami. ne on prolactin release. Endocrinology 88: 1012, 1971.

21. FLUCKIGER E, del POZO E, VONWERDER K: Prolactin: Physiology, Pharmacology and Clinical Findings. Berlin, Springer Verlag, 1982.

22. PARKER DC, ROSSMARIN LG, VAN DER LAAN EF: Sleep related, nyctohemeral and briefly episodic variation in human plasma prolactin concentration. J Clin Endocrinol Metab 36: 1119, 1973.

23. LUCIANO AA, CHAPLER FK, SHERMAN BM: Hyperprolactinemia in poly cystic ovary syndrome. Fertil Steril 41: 719, 1984. 